\title{
PROKLA-Redaktion
}

\section{Akteure beim Hochseilakt mit Netz, ein Experiment}

Für viele Gesellschaften, die im vergangenen »neoliberalen Jahrzehnt « ihre Entwicklungshoffnungen auf die Dynamik eines entfesselten Marktes gesetzt haben, hat sich dieses Projekt als Tanz auf dem Drahtseil ohne Netz herausgestellt. Besonders tief war der ungesicherte Absturz in den »Transformationsgesellschaften « Ost- und Mitteleuropas, wo die marktliberalen Schocktherapien im Stile der Strukturanpassungsprogramme des IWF oder marktliberaler Sequenzprojekte mit wenigen Ausnahmen (in kleinen und bereits vergleichsweise entwikkelten zentraleuropäischen Ländern) gescheitert sind. Aber schon lange vor den Erfahrungen in Osteuropa zeigte sich in vielen lateinamerikanischen Ländern, daß das bloße Setzen auf den Markt nicht nur ökonomisch unzureichend ist, sondern auch zur Veränderung politischer Institutionen wie auch des »Politischen « selbst führt. Das Gegenwärtige wird »omnipräsent« und die Fähigkeit zur Gestaltung der Zukunft geht verloren (so Norbert Lechner in seinem Beitrag).

Inzwischen ist der theoretische Befund überzeugend und auch politisch weitgehend akzeptiert, daß Strategien der
Öffnung, also der Integration in den real existierenden Weltmarkt, institutionelle Voraussetzungen haben, die nicht einfach in kurzer Frist nach einem in den IWF-Labors entwickelten Rezeptbuch erzeugt werden können. Es genügt nicht, alte Regularien einzureißen und Marktkräfte $»$ freizusetzen«, es müssen auch Institutionen erzeugt und dann gepflegt werden, die es den Marktakteuren überhaupt erst ermöglichen, »effizient« zu handeln. Soll sich der Markt nicht von vornherein als bloß destruktive »Teufelsmühle (Karl Polanyi) erweisen, so müssen gesellschaftliche Bindungen geknüpft werden, in denen die Marktakteure mit ihrer bloß mikroökonomischen Rationalität eingebettet sind. Diesem Thema, auf das Karl Polanyi schon vor Jahren aufmerksam machte, und das nichts von seiner Aktualität eingebüßt hat, ist die vorliegende Ausgabe der PROKLA gewidmet.

Die Debatte um Wettbewerbsfähigkeit von »Standorten «, die weltweit höchst polemisch geführt wird (vgl. dazu den Beitrag von Elmar Altvater), hat gerade entgegen dem verbreiteten $\gg$ Marktfundamentalismus ( vgl. dazu Stephan Elkins in PROKLA 96, September 1994) 
zu Tage befördert, daß ökonomische Effizienz und Erfolge nicht nur von den Allokations- und Distributionsmechanismen von Märkten abhängen oder der Kompetenz regulierender Interventionen des Staates geschuldet sind, sondern sozialer, nicht-marktförmiger Netze bedürfen. Neben der unsichtbaren Hand des Marktes und der sichtbaren Hand des Staates gibt es noch die von Diane Elson in PROKLA 78 (März 1990) so bezeichnete »dritte Hand« gesellschaftlicher Institutionen. Sie können den Akteuren auf Märkten Informationen vermitteln, Sicherheiten geben, Vertrauen erzeugen, auf dem die einzelnen Tauschakte zwischen Käufer und Verkäufer auf dem Markt aufbauen. So können Transaktionskosten vermindert und Unternehmen auf den Weltmärkten »schlagkräftiger werden. Die »soft factors« der Wettbewerbsfähigkeit können aber - anders als Marktkräfte nicht kurzfristig »erzeugt« werden, sie haben eine je spezifische Geschichte. Netzwerke, institutionelle Verflechtungen wachsen quasi-organisch als eine gesellschaftliche und ökonomische Produktivkraft. Die Bedingungen der Entstehung und Festigung solcher Netzwerke nicht-marktförmiger Regulation wurden auch schon in früheren Heften der PROKLA untersucht (vgl. die Beiträge von de Vroey, Semmler, Schwengel, Bugaj/Kowalik und Müller-Plantenberg in PROKLA 82, März 1991). Allerdings können sich diese Netzwerke unter spezifischen Umständen auch in Innovationshemmnisse, in Blockaden von Effizienz und Evolution verwandeln. Neben den vieldiskutierten Phäno- menen von Staats- und Marktversagen lassen sich ebenso Formen des »Netzwerkversagens« beobachten und theoretisch begründen (vgl. dazu den Beitrag von Dirk Messner).

Es ist auch fraglich, ob die Propagierung und Unterstuitzung von nicht-marktförmigen Netzwerken die Zukunftsfähigkeit von Gesellschaften tatsächlich befördert, wenn sie lediglich zur Hebung der regionalen und nationalen Konkurrenzfähigkeit auf dem Weltmarkt dienen sollen. Netzwerke können dann sehr schnell als soziales Korsett der gesellschaftlichen Formierung instrumentalisiert werden - wohlstandschauvinistisch gegen andere Regionen und Nationen. In der neuen »Geoökonomie $\ll$ kann der Staat zum »Wettbewerbsstaat« (Joachim Hirsch) werden, der die Netzwerke als Instrumente zum Einfangen der aus einem »Produktivitätspakt« ausscherenden gesellschaftlichen Kräfte nutzt. Freilich bilden sich im Zusammenspiel der Artikulation von Marktbeziehungen, staatlich-hierarchischer Interventionen und gesellschaftlichen Netzwerken komplexe »Akteursarenen« und »Handlungstrajektorien $\ll$ heraus. Die daraus resultierende gesellschaftliche Entwicklungsdynamik ist weder durch Marktmodelle realistisch modellierbar, noch gemäß einer Taxonomie des Staatshandelns leicht einzuschätzen. Theoretisch komplexere Ansätze sind, wie Dirk Messner aufzeigt, gefragt.

Die »Netzwerkanalyse« wird freilich auch über den eher ökonomischen Anwendungsbereich hinaus fruchtbar $\mathrm{zu}$ machen versucht. Institutionelle Netzwerke der »Politikverflechtung « zwi- 
schen verschiedenen Entscheidungsebenen und Akteursarenen sind ebenso von Bedeutung, wie neue Formen der Vernetzung von Nicht-Regierungsorganisationen (dazu der Beitrag von Achim Brunnengräber und Heike Walk). Angesichts der begrenzten materiellen Mittel, die NGO's zur Verfügung stehen, kann Vernetzung zu einer dieses Defizit kompensierenden Ressource werden. Weltweite informationelle Vernetzung hat die Schlagfertigkeit von NGO's enorm gesteigert, wie während der UNCED-Konferenz vor 1992 deutlich wurde, und wie es in den Vorbereitungen der Weltklimakonferenz in Berlin im Frühjahr 1995 erneut versucht wird.

Ganz anders wiederum stellt sich Vernetzung »offizieller« international wirksamer Institutionen dar, wie der Beitrag von Wolfgang Zaschke zeigt. Wenn »der« Sozialstaat als Nationalstaat in der Krise ist und gleichzeitig der Sozialraum durch Migrationsbewegungen und die Attraktivität des westlichfordistischen Akkumulationsmodells ungebrochen ist und daher eine unbestritten internationale Dimension aufweist, werden auch von der Internationalen Arbeitsorganisation neue $» v e r n e t z-$ te « Reaktionsformen auf die neuen Herausforderungen verlangt.

Netzwerke - das sind höchst unterschiedliche institutionelle und funktionelle Bindungen von ganz verschiedenen Akteuren mit divergenten Handlungslogiken. Die Kategorie verweist auf die Bedeutung des »in between «, des auch theoretisch lange vernachlässigten Raums zwischen Markt und
Staat, auf die $»$ Kraft loser Bindungen $\ll$ in gesellschaftlichen Evolutionsprozessen, auf eine soziale Dimension der »Strukturierung « (Giddens) von Systemen durch Handeln von Akteuren, das nicht einer festen (markttheoretisch begründbaren oder taxonomisch bewertbaren) Handlungslogik folgt, aber auch nicht bloßes Experiment in einem völlig offenen trial-and-error-Prozeß 3 ist. Solange die Akteure auf den Drahtseilen des Marktes agieren und stets mit dem möglichen Absturz konfrontiert sind, werden sie ohne solche Netze nicht auskommen.

In einem Beitrag außerhalb des Heftschwerpunkts setzt sich Sabine Lang mit der sich gerade erst etablierenden Geschlechterforschung in der Politikwissenschaft auseinander. 